\title{
Optoelectronic Devices Employing One-Dimensional Photonic Structures
}

\author{
J. Muszalski \\ Instytut Technologii Elektronowej, al. Lotników 32/46, 02-668 Warszawa, Poland \\ This paper reviews the experimental and theoretical results ob- \\ tained during work on the modern semiconductor devices employing one- \\ dimensional photonic structures. After short review of the physical features \\ of structures consisting of $1 \mathrm{D}$ stack of the alternating high and low index \\ layers, particular attention will be given to unique features of the devices \\ employing microcavities: resonant cavity LEDs, resonant-cavity enhanced \\ photo-detectors, vertical cavity surface emitting lasers, and also vertical ex- \\ ternal cavity surface emitting lasers. At the end the semiconductor saturable \\ absorber mirrors are discussed. \\ PACS numbers: 73.21.Fg, 73.40.Kp, 78.20.Ek, 78.40.Fy, 78.47.Cd, \\ 78.55.Cr, 78.60.Fi, 78.67.De
}

\section{Introduction}

The optoelectronic devices require precise control of both carriers and photons. The modern semiconductor technology which allows for fabrication of thin layers laterally engraved gives such opportunity. The single nanometers distances provide the confinement for the carriers whereas the tens of nanometer thick ones permit to engineer the light propagation. The difference in the scale comes directly from the corresponding wavelengths difference: de Broglie wavelength of the carriers and the wavelength divided by the refractive index of the light. Since the refractive index contrast is rather low for most materials the light confinement has often to be enhanced by periodic modulation of the refractive index. This allows for modification of the light propagation in similar way as a periodic potential does in solid state. Such periodic structures are called photonic crystal. Depending of the application and available technology the photonic crystal can be one-, twoor three-dimensional. The $1 \mathrm{D}$ photonic crystals are fabricated most of all as a stack of high and low index layers. The technology of thin layers is well developed for many materials both dielectric and semiconducting. The $2 \mathrm{D}$ photonic crystals are realized by engraving a pattern on an active surface. Since the characteristic length of the pattern has to be comparable to the light wavelength for the visible 
and infrared spectral range the pattern has to be engraved with a sub-micrometer precision. This is feasible with focused electron beams. The 3D photonic crystals are the most desired, they are realizable by combining the thin layers stacks with the laterally engraved patterns.

However, a practical realization of photonic crystal which has found numerous applications in devices, is a stack of semiconductor layers epitaxially grown by molecular beam epitaxy (MBE) or metal organic chemical vapor deposition. The semiconductor epitaxial films of different chemical composition differ by the refractive index and at the same time by the electronic band gap. This allows, for instance, to capture at the same time carriers and the photons in the same space and efficiently control their coupling.

\section{1D photonic crystal}

As an example of such 1D photonic crystal let us consider a stack of GaAs/AlAs layers. Such stack can be quite easily epitaxially grown. The refractive indexes of GaAs and AlAs are 3.5 and 2.94, respectively [1]. Thus the contrast is 0.56 . Periodic modulation of the index in such stack results in photonic band gap, the so-called stop-band (Fig. 1a). The spectral position of the stop-band corresponds to the half wavelength $\left(\lambda / 2 n_{\mathrm{R}}\right)$ optical thickness of the GaAs/AlAs layer pairs. Although the spectral position is determined by the total optical thickness of the high and low index layers the lowest transmission is when optical thicknesses are quarter wavelength ones $\left(\lambda / 4 n_{\mathrm{h}}, \lambda / 4 n_{\mathrm{l}}\right)$ i.e., optically equal for both layers. In case of non-absorbing materials the low transmission translates directly to high reflectivity. Indeed, the quarter wavelength thick stack called also distributed Bragg reflectors (DBR) are widely used due to their high reflectivity. The high reflectivity of the DBRs comes from collective reflections of the light on the subsequent interfaces. The quarter wavelength thickness change the phase by $\pi / 2$ of forward and after reflection backward traveling waves in consequence the amplitude of reflected wave builds-up by positive interferences. Because of that it is obvious that the refractive of DBR depends on the number of layers pairs and the index contrast

$$
r_{\mathrm{DBR}}=\frac{1-\left(n_{\mathrm{s}} / n_{\mathrm{o}}\right)\left(n_{1} / n_{2}\right)^{2 m}}{1+\left(n_{\mathrm{s}} / n_{\mathrm{o}}\right)\left(n_{1} / n_{2}\right)^{2 m}},
$$

where $\mathrm{s}$ and o are the indices of the adjacent layers, substrate and most often air $[2]$.

For elevated number of pairs the reflectivity can approach unity, that is, it can be much greater than that of the best metal mirrors. The reflection of the light wave by a DBR results in the formation of standing wave. The characteristic feature is that both the nodes and antinodes are located exactly at the interfaces (Fig. 1b). The node is when the light passes from the low index layer to the high index one, and the antinode when it passes from the high to the low index layer.

At the wavelength shorter and longer than the photonic band gap the light propagates along the photonic crystal. The short wavelength range adjacent to 

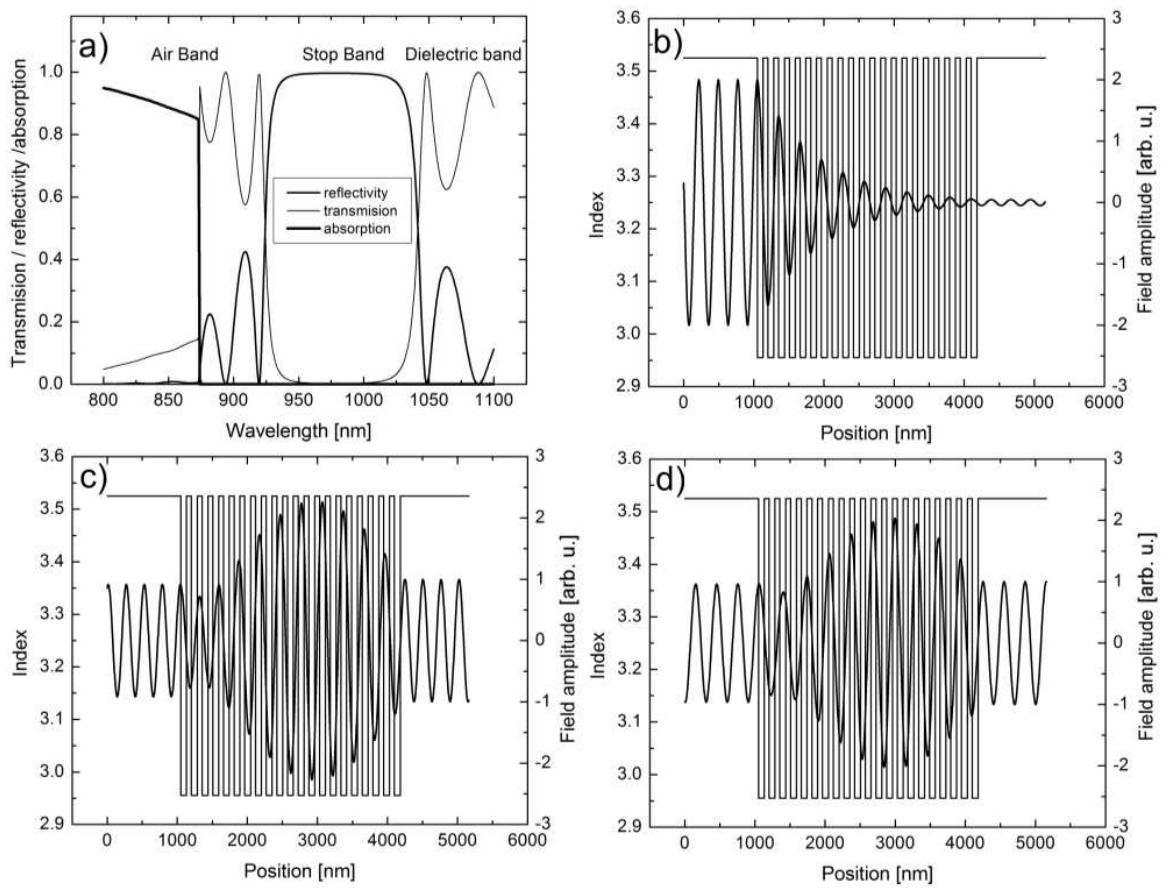

Fig. 1. The spectral bands of a one-dimensional photonic crystal consisting of a stack of high GaAs and low AlAs index layers (a), the electromagnetic field distribution along the heterostructure at the resonant wavelength $980 \mathrm{~nm}$ (b), at the air band $920 \mathrm{~nm}$ (c), at the dielectric band $1050 \mathrm{~nm}$ (d). For the wavelength shorter than $870 \mathrm{~nm}$ the absorption in GaAs dominates the light propagation.

the stop-band is called air band whereas the long wavelength the dielectric band. The names of those bands came from the characteristic distribution of the electromagnetic field of propagating waves. At the air band the antinodes are located in the center of the low index layer (Fig. 1c), in contrast to the dielectric band where the antinodes take place in the high index layers (Fig. 1d). In addition, in those bands the amplitude of electromagnetic field distribution is modulated. This is because the average index of DBR is different than the adjacent media. In consequence the DBR volume constitutes a cavity whose resonances modify the electromagnetic field distribution.

Both the transition of the light and the electromagnetic field distribution can be easily modeled by the transfer matrix method [2]. This formalism especially suitable for computer calculation, associates with each interface two matrixes $2 \times 1$ which describe the amplitude of the forward and backward traveling waves on both sides of this interface. The phase factor describes the change in the phase of the light traveling through a layer. A simple multiplication of those matrixes allows for the amplitude and the phase calculation for each point along the photonic crystal. 
In a $1 \mathrm{D}$ photonic crystal the refractive index is modulated only along one direction ( $z$ axis), so when the light is aimed not perpendicularly to the surface only a single component $k_{z}$ of propagation constant $k$ interacts with the photonic crystal. The propagation constant itself $k=2 \pi n_{\mathrm{R}} / \lambda$ is inversely proportional to the wavelength thus when the angle is increased the resonant wavelength is red shifted. This red-shift is described by

$$
\lambda_{(\theta)}=\frac{2 L}{m} \sqrt{n^{2}-\sin ^{2}(\theta)},
$$

which is a product of the Snell law and the propagation $2 k L \cos (\theta)=2 m \pi, m$ is the cavity order, $L$ - its thickness, $n$ - index of cavity material and $\theta$ - the propagation direction outside the cavity.

For the light aiming at the surface not perpendicularly one also has to distinguish between the TE and TM polarized waves.

The features of semiconductors depend on the potential of the ion cores, the greater potential the wider band gap. In a similar way the photonic band gap depends on the refractive index contrast. Greater index contrast results in the wider stop-band. The refractive index contrast puts the same constraints for the applications, as for instance, presented below modulators require wide stop-bands, in contrast to spectrally selective photodetectors which gain from narrow ones.

The analogy between the photonic and the electronic structures goes even further. As in semiconductor structure a defect in periodicity or a foreign atom gives an additional level in the band gap, a layer of different refractive index or different thickness results in the additional state in the stop-band. The light is trapped on this defect as the carriers on the dopant. The limit of this analogy is however the Fermi-Dirac statistic which applies to the carriers in the solid state and does not to the light waves in photonic crystal. The difference lies also in a limited number of layer pairs in comparison to the immense number of the ion centers in solid state.

A single out of period layer, i.e., a half wavelength thick in the quarter wavelength stack of the alternating high and low index ones results in the transmission band in the center of the stop-band (Fig. 2). The exact spectral position of this transmission band is a function of the out of order layer thickness. If the thickness of any chosen layer is increased from a quarter wavelength the band appears on the short wavelength edge of stop-band and reaches the center when the layer thickness is exactly half wavelength. Further increase in the layer thickness redshifts the transmission band. For the layer thickness equal to the three quarters the high reflectivity is restored over the whole stop-band. The three-quarter wavelength thickness changes the phase of traveling light as a single-quarter one, it does not disturb the periodicity of the stack. Further increase in the thickness of the considered layers repeats the red shift of the transmission band from short to long wavelength edge of the stop-band up to the point when the second, third, etc. transmission band appears. 

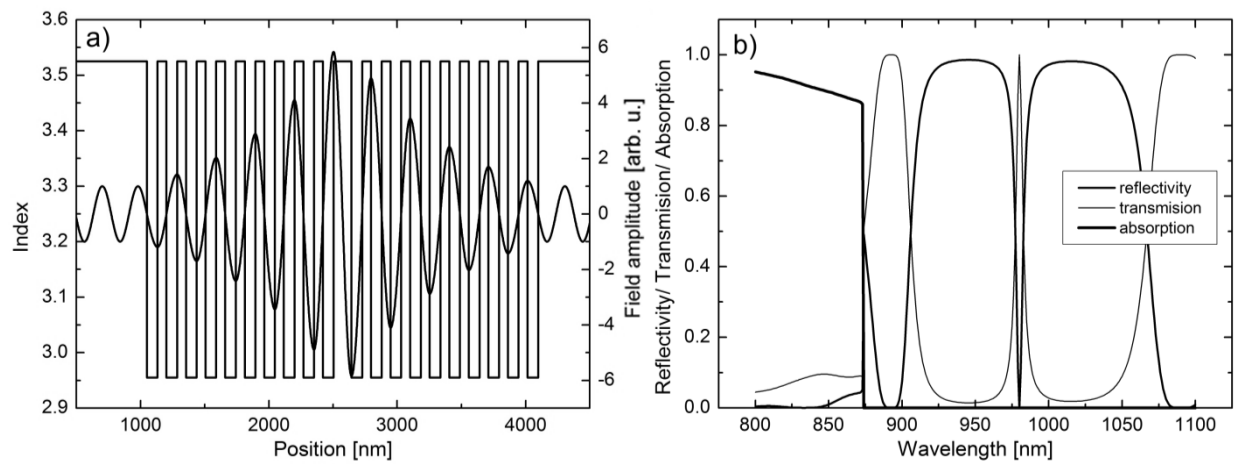

Fig. 2. One-dimensional photonic crystal consisting from a stack of quarter wavelength GaAs/AlAs layers (a). A single AlAs layer is missing which constitutes a defect in the crystal structure. Transmission and reflectivity of such photonic crystal (b).

Any chosen layer, when its thickness is different from the quarter wavelength one, shall be considered as an optical cavity enclosed by two reflectors. The review on the cavities properties can be found in [3-6]. The layer thickness determines the cavity order. Greater thickness, greater cavity order. For elevated cavity orders when the resonances are close enough. i.e., the free spectral range is smaller than the stop-band, greater number of the transmission lines is observed.

In case of the cavity enclosed by a DBR the cavity order is enlarged by the penetration depth of the mirrors (see Fig. 1b)[3]. The penetration depth is a very important parameter describing a DBR. When the light is refracted by a DBR it changes the phase. This is especially pronounced, when the light is aimed not perpendicularly to the surface. This change of the light phase can be very well described as it would be reflected by a mirror located further not exactly on the DBR surface. This distance is called a penetration depth and describes the real penetration of the light which is especially deep in case of low refractive index contrast DBR.

The optical cavity features are described by the finesse and quality factor. The greater reflectivity of the mirror the slimmer transmission band, but also greater electromagnetic field amplitude in the cavity. The augmentation of the electromagnetic field is the main feature of resonant cavity which have found application in semiconductor devices.

\section{Photo-detectors}

The very strong augmentation of the electromagnetic field in a cavity enclosed by two DBR induce the photo-detector application. Such devices are referred as resonant-cavity enhanced photo-detectors (RCE-PD) [7]. The high field amplitude allows for effective absorption on the thin layers thus fabrication of the broad bandwidth devices. The higher finesse of the cavity the thinner absorber 
can be. The price for it is however the spectral response shrinkage. Therefore the medium finesse cavities have found the widest application in photo-detectors.

An exemplary device is a photo-detector optimized for backside operation at $1.55 \mu \mathrm{m}$. Since the refractive index contrast for the InP lattice match InAlAs and InGaAs is low the RCE-PD requires elevated number of the high/low index layer pairs, the most noble example of such device is in Ref. [8]. Here an alternative heterostructure was proposed and fabricated [9]. The epitaxial growths were performed with MBE Riber 32P system. Lattice matched epitaxial layers of $\mathrm{In}_{0.53} \mathrm{Ga}_{0.47}$ As and $\mathrm{In}_{0.52} \mathrm{Al}_{0.48}$ As were grown on (001) oriented InP substrates. The detector structure itself was grown on the $\mathrm{In}_{0.52} \mathrm{Al}_{0.48} \mathrm{As}: \mathrm{Si}$ buffer layer deposited directly on InP. Since the discontinuity of the refractive index at the substrate buffer interface is part of photonic structure, the thickness of the buffer layer was $2287 \mathrm{~nm}(2 \lambda)$ thick. This layer was heavily Si doped. This layer was followed by DBR which consisted of 7 pairs of $\left(\mathrm{In}_{0.52} \mathrm{Al}_{0.48} \mathrm{As} / \mathrm{In}_{0.53} \mathrm{Ga}_{0.47} \mathrm{As}\right): \mathrm{Si}$ designed for high reflectance at $1.55 \mu \mathrm{m}$. The doping concentration in DBR layers was equal to $10^{19} \mathrm{~cm}^{-3}$. It results in the red shift of the absorption edge of the $\operatorname{In}_{0.52} \mathrm{Al}_{0.48} \mathrm{As}$ and especially $\mathrm{In}_{0.53} \mathrm{Ga}_{0.47}$ As layers beyond the spectral range of interest. This is due to the Burstein effect associated with conduction band filling with carriers. A $140 \mathrm{~nm}$ thick $\mathrm{In}_{0.53} \mathrm{Ga}_{0.47}$ As absorber was placed in between two $\mathrm{In}_{0.52} \mathrm{Al}_{0.48} \mathrm{As}$ spacer layers of the thickness $283 \mathrm{~nm}$ and $493 \mathrm{~nm}$, respectively. The asymmetrical cavity was designed to: first, to place the absorber in the antinode position of the resonant wave, and second, to facilitate the intra cavity contact fabrication. The $p$-type doping was lower $\left(2 \times 10^{18} \mathrm{~cm}^{-3}\right)$ at the proximity of the absorption layer in order to prevent the beryllium back diffusion and higher $\left(10^{19} \mathrm{~cm}^{-3}\right)$ at the heterostructure surface. After epitaxial growth on the top surface a hybrid mirror: consisting from dielectric and metallic was deposited in order to enclose the cavity. Dielectric reflectors were formed by deposition quarter-wavelength stacks of $\mathrm{SiO}_{2}$ and $\mathrm{Si}_{3} \mathrm{~N}_{4}$. Numerically simulated distribution of electromagnetic field across structures studied equipped with metallic and hybrid (dielectric DBR+metal) reflectors on the top of the structures is presented in Fig. 3.

Since in the case of epitaxial growth of DBR the very high precision of the grown layer thickness is required, the special attention was paid to the growth rate control. In addition to the calibration done prior to the growth, the DBR runs were controlled in situ using the pyrometric interference technique [10-12]. The pyrometric interference technique is a very convenient alternative to the most commonly used in situ refractometry or elipsometry. Its idea is based on the simple fact that in the case of heterostructures growth the layers of different composition have different optical constants and as a result the thermal radiation generated in the substrate undergoes multiple reflections at the internal interfaces and the surface of growing structure. Consequently, the various reflected waves interfere. This interference can be observed using narrow band width pyrometer. The oscillations of the pyrometric signal are the function of the growing layer thickness. 


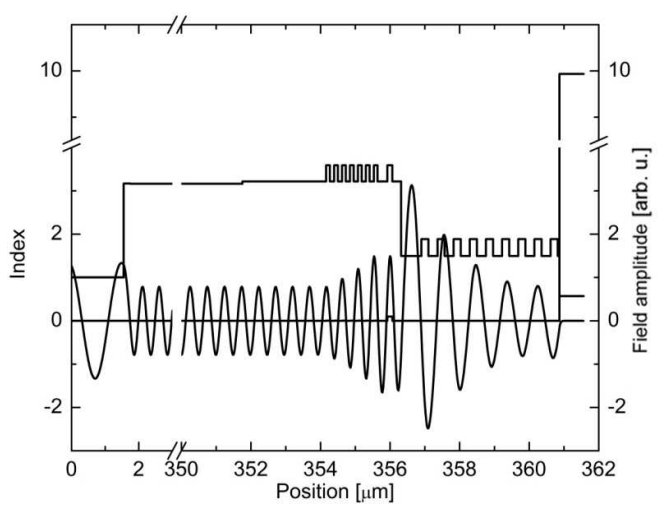

Fig. 3. Refractive index distribution along a RCE-PD heterostructure, distribution of electromagnetic field is also shown.

The phase and the period of oscillations provide information on the actual growth rate. The oscillations amplitude can be as high as $15^{\circ} \mathrm{C}$. Although this does not permit the instant temperature measurements, the mean value taken over a long time can be correlated with the true substrate temperature.

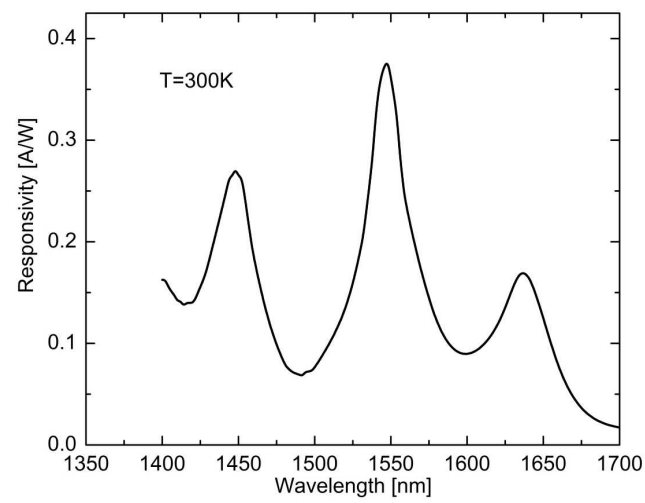

Fig. 4. Spectral response of a RCE-PD [9].

During the device fabrication session first a number of test heterostructures has to be grown in order to calibrate the materials growths. The InAlAs, InGaAs epitaxial layer compositions were determined from X-ray diffraction studies using diffractometer in the double and triple axis configurations. Carrier concentrations were determined from the Hall effect studies performed on test layers. The dielectric layers were deposited in plasma enhanced chemical vapor deposition (PECVD) process. The dielectric reflectors consisted of $324.18 \mathrm{~nm} \mathrm{\textrm {SiO } _ { 2 }}$ layer followed by 9 pairs of $259.18 \mathrm{~nm}$ thick $\mathrm{SiO}_{2}$ layers and $205.40 \mathrm{~nm}$ thick $\mathrm{Si}_{3} \mathrm{~N}_{4}$ layers. The devices were fabricated by standard photolithography process. Ohmic contacts to top $p^{+}$layers were formed by Ti-Pt-Au lift-off technique. All layers down 
to $\mathrm{Si}$ doped $\mathrm{In}_{0.52} \mathrm{Al}_{0.48}$ As were etched away using isolation mask, forming mesa structure with $300 \mu \mathrm{m}$ diameter. Ohmic contacts to $n^{+}$layers were formed by $\mathrm{Au} / \mathrm{Ge}-\mathrm{Ni}-\mathrm{Au}$ lift-off. The fabricated devices were tested at room temperature and backside illumination. Photoresponse measurements were carried out in the 1350-1700 nm range. The light was directed at normal incidence to the device in exception of measurements of incidence angle dependences. The photoresponse of $2 \lambda$ RCE $p-i-n$ PDs is shown in Fig. 4. The enhancement of the quantum efficiency for the resonant wavelength is strongly pronounced by an absorption peak centered around $1550 \mathrm{~nm}$. Its full width at half-maximum (FWHM) is equal to $30 \mathrm{~nm}$. Two side absorption lines outside the DBRs stop-bands were detected at about $1450 \mathrm{~nm}$ and $1650 \mathrm{~nm}$ wavelength. The responsivity of the side peaks is lower by $30 \%$ and $50 \%$, respectively.

\section{Microcavity employing emitters}

In analogous way the parameters of a microcavity enclosed devices are modified in case of emitters. When the light source is placed in a cavity it is exclusively coupled to the cavity modes. This allows for spectrally shrunk and amplitude enhanced emission [13]. A practical realization of such device is a resonant cavity enhanced light emitting diode (RC LED). Such diode was for the first time realized by Schubert et al. [14], and later by others [15-17]. One of such realization is a device designed for the emission at $1000 \mathrm{~nm}$ in which the $8 \mathrm{~nm}$ thick InGaAs quantum wells located in a GaAs slab are enclosed by two DBR [18]. The number of high GaAs and low AlAs index layers pairs was 15 and 8 for the substrate and surface side DBR, respectively. Placing the $p-n$ junction in the slab center assures efficient injections of the carriers and their radiative recombination in quantum wells. Since the GaAs is a high index material at the resonant wavelength there are antinodes at the cavity DBR interfaces. On the other hand, in order to allow the emission perpendicularly to the surface, along the growth direction, the quantum wells (QWs) have to be located also in the antinode position. To fulfill both those constraints the smallest cavity has to be $\lambda$ type. The $\lambda$ type microcavity is the order two cavity. However, the penetration depth of the AlAs/GaAs DBR slightly excides $(3 / 2) \lambda[3]$. In consequence the total order of such microcavity is equal to 8 . This means that the cavity supports 8 modes: in equal number symmetric and antisymmetric. Only symmetric modes have antinodes in the center of the cavity, i.e., on the QWs. Thus only to those four modes emitted light is coupled. The mode for which the propagation vector is along the growth direction can be totally extracted. This makes the extraction efficiency of the RC LED equal to $25 \%$ which shall be compared with $2 \%$ for standard surface emitting LED. For other modes the propagation vector lies outside the extraction cone [13] so they can be extracted only by the chip edge.

The RC LED structures studied in this work were fabricated by solid source MBE. In practical realization of the RC LED in order to minimize series resistance 
of the DBR mirror the grading of the interfaces composition is required. Here as an example of such grading between the GaAs and AlAs layers $20 \mathrm{~nm}$ thick $\mathrm{Al}_{0.5} \mathrm{Ga}_{0.5} \mathrm{As}$ layer was introduced (Fig. 5) [6].

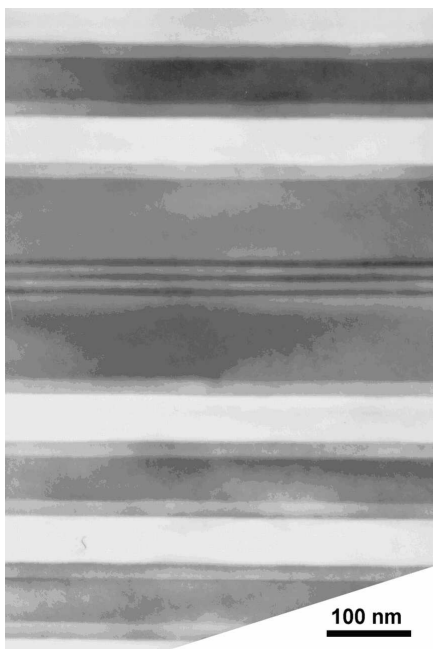

Fig. 5. The cross-section of a RC LED. The most dark lines correspond to the InGaAs QW, the gray to the GaAs and light gray to AlAs. On the GaAs/AlAs interfaces thin $5 \mathrm{~nm}$ AlGaAs layers grading the band-gap discontinuity is well visible [6].
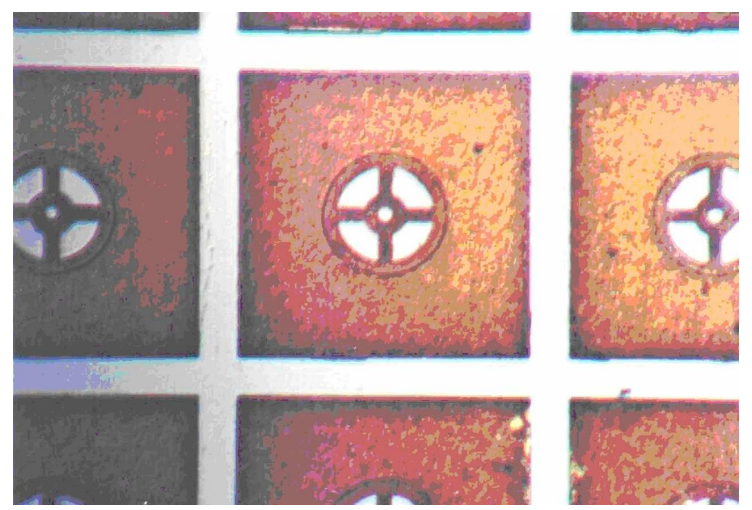

Fig. 6. A matrix of ready to use RC LED [6].

The RC LED characterizes by surface emission which is an advantage. The devices can be fabricated as matrixes which allows to scale up the device power (Fig. 6). An advantage is that they can be tested at early stage of the fabrication process before the cleavage to the individual chips. Here the diodes were fabricated by conventional photo-lithography and metallization process. The light from the 


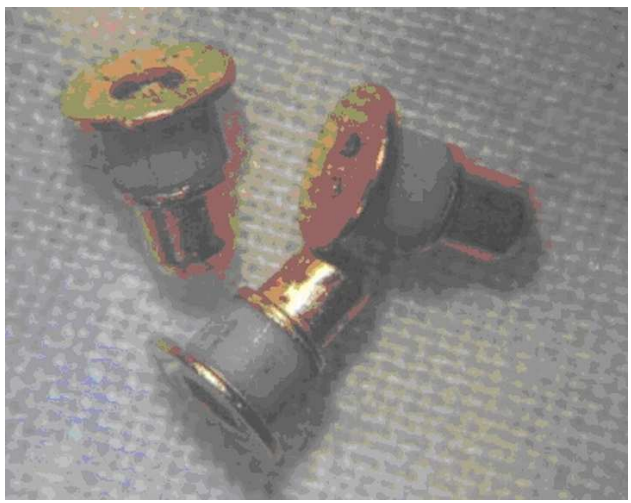

Fig. 7. The individual chips mounted in microwave cases [6].
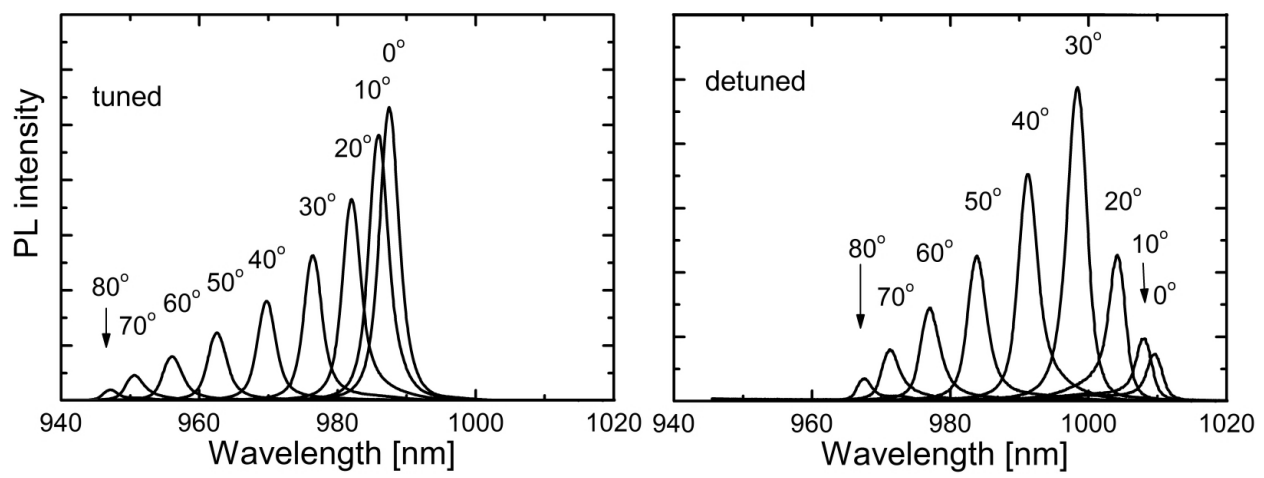

Fig. 8. Angle dependent emission of RC LED [6].

diode was extracted through the openings in the upper $\mathrm{Cr}-\mathrm{Pt}$ contact. The bottom $\mathrm{Au}-\mathrm{Ge}-\mathrm{Ni}$ contact (to the $n$-type substrate) formed a solid circle. The diodes were electrically tested at probe tester and good ones were assembled in high frequency microwave type cases (Fig. 7).

The emission from QWs located in a micro-cavity is strongly modified by the cavity [19]. At given direction of observation the emission is spectrally poor and strong (Fig. 8). This is a consequence of the coupling to the cavity modes. The observed enhancement of the amplitude comes from the fact that the light at given wavelength is emitted only in one direction (strictly in a cone because of the cylindrical symmetry) which is in contrast to the standard LED where a QW emits light at all wavelengths in all directions. The spectral width of electroluminescence line depends on the cavity finesse and can be as low as $1 \mathrm{~nm}$ for high finesses cavities. This is however observed only when the photoluminescence (PL) signal is collected in the sufficiently narrow solid angle. When the light is collected in wide solid angle, the observed PL lines are substantially broadened as is shown by Eq. (2). 
Designing the RC LED for maximum extraction efficiency one has to take into account the spectral width of the light source. The full width at half maximum of the emission of a InGaAs/GaAs QW is typically $20 \mathrm{~nm}$ at low excitation. This is much more than the FWHM of even low finesse cavity. For perfectly tuned cavity resonance - QW emission - the light at the wavelength longer than the resonance cannot be extracted. In order to extract maximum of the light a certain detuning of the QW emission and the cavity resonance is required. The QW should be blue-shifted in respect of the cavity resonance (Fig. 9) [20]. Then the maximum emission goes not perpendicularly to the surface but at certain angle, in a cone (Fig. 10) [21].

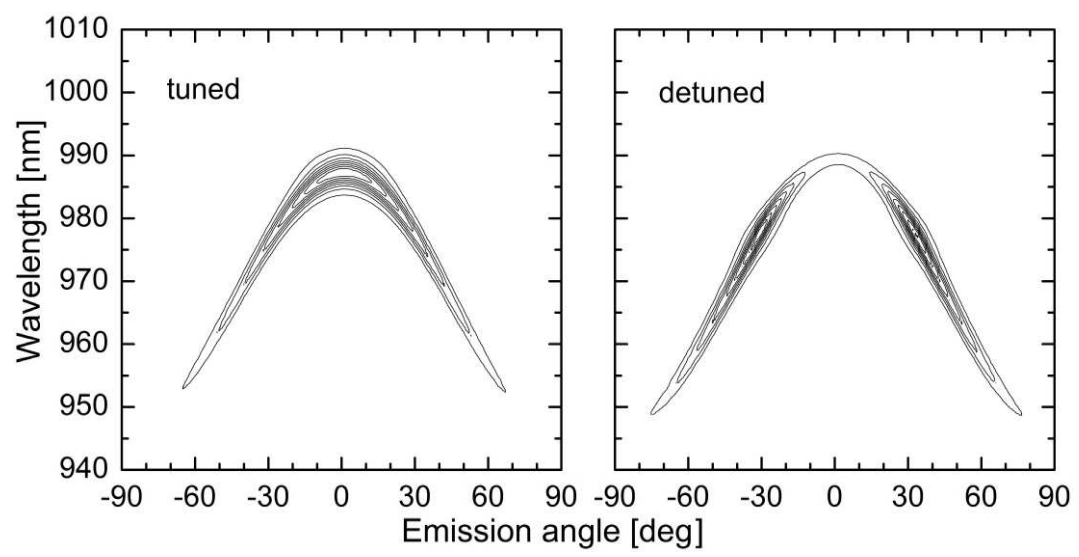

Fig. 9. The electroluminescence intensity as a function of both the wavelength and emission angle. Data for well tuned and slightly detuned devices [19].

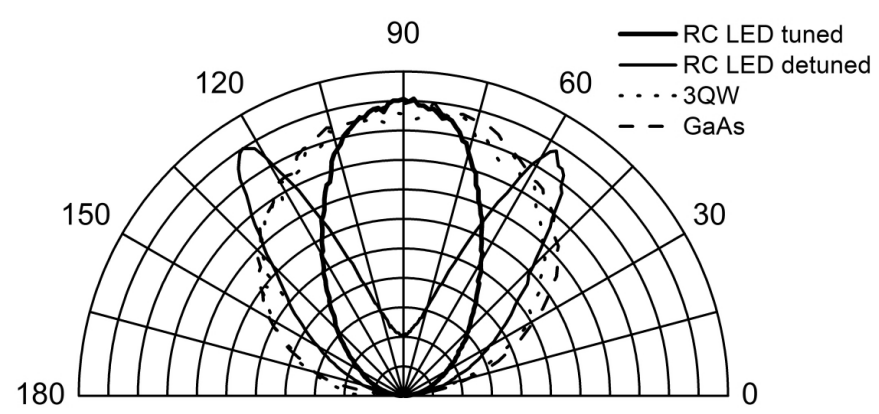

Fig. 10. The integrated emission of tuned (single lobe) and slightly detuned (twin lobe) devices [6].

The features which give the RC LED an advantage over other LED designs is a very small temperature dependence on the device temperature $[22,23]$. The temperature drift of emission wavelength in standard LEDs reflects the change of 
the active region band gap with temperatures. This drift in case of the RC LEDs is strongly suppressed from $2.4 \mathrm{~nm} / \mathrm{K}$ to $0.1 \mathrm{~nm} / \mathrm{K}$. The emission wavelength of a RC LED depends on the spectral position of the resonance. The resonance itself depends on the geometrical parameters - the layer thicknesses of the heterostructure and the layer indices. Both those parameters are much more stable than the band gap. The different temperatures drifts of the resonant wavelength and the QW emission can be used in the tuning investigation [19]. By careful change of the temperature a detuned device can be tuned and its potential estimated (Fig. 11) [24].

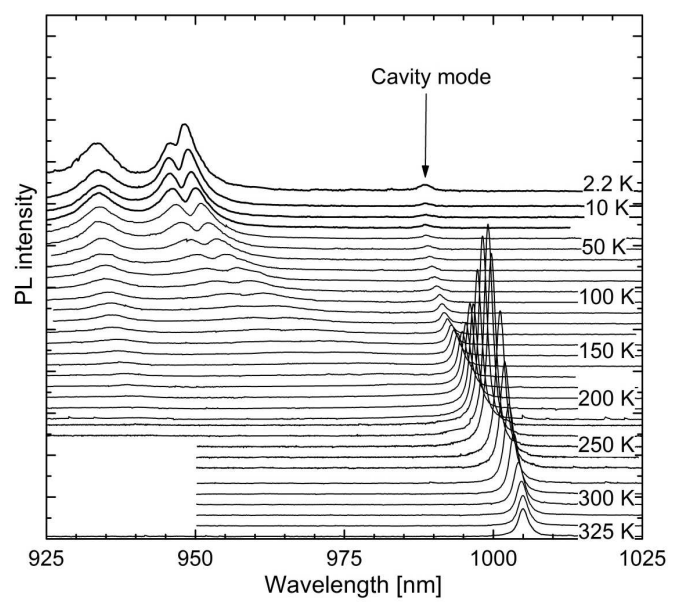

Fig. 11. The temperature tuning of a RC LED. The difference in the temperature drift of a cavity resonance and the QW peak emission allows to tune the device and evaluate its design potential [16].

RC LED due to their high efficiency have found application in optical interconnection. The small distance communication based on plastic optical fibers (POF), for instance, in car or aircraft where the interconnections of the sensors but also multimedia have to be resistant to electromagnetic noise. The small power, temperature independent, thresholdless RC LED are an alternative for more sophisticated laser based systems.

\section{Vertical cavity surface emitting lasers}

The low finesse cavities of RC LED allows for spontaneous emission engineering. When the reflectivity of DBR mirrors is increased to high values approaching almost unity the round trip losses became small and at high excitation the threshold of a stimulated emission can be overpassed. This takes place in the VCSEL and VECSEL (acronyms for vertical cavity surface emitting laser [25-27] and vertical external cavity surface emitting laser [28, 29]). Those surface emitting lasers differ only by top mirror. In VCSEL it is integrated with the active region in a single 
epitaxial process. In VECSEL the top mirror is a concave mirror mounted at certain distance from the chip. Both constructions have different advantages. The VCSEL offer a relative simplicity of fabrication in single epitaxial run, excellent beam quality suitable for efficient fiber coupling, high band-width of modulation. Those advantages make the VCSEL a device of choice for optical fiber communication. The only disadvantage of the VCSEL is the limited power. This limitation originates in small thickness of the active region. The insufficient spread of carriers from a ring electrode used for the light extraction, does not permit uniform excitation of a gain medium over large area. Also the very high excitation of small VCSEL leads to the degradation of the device performance. First, due to the rapid temperature rise due to the high heat flux density, and latter, due to the catastrophic optical mirror damage.

The low power limit can be overcome when in state of electrical pumping the optical excitation is applied. There is no spatial limit for uniform excitation of the semiconductor surface laser medium. Nowadays the high power edge emitting lasers are accessible. They can be used to pump the surface emitting lasers. In such approach the high power but low beam quality emission of the edge emitting laser is transformed with high efficiency to the single lateral mode $\mathrm{TEM}_{00}$, highly coherent, low divergent cylindrical beam.

We have grown both the VCSEL and VECSEL heterostructure. Those heterostructures consisted of GaAs/AlAs DBRs and the InGaAs QWs designed for the emission in the 980-1000 nm band. Both devices were designed for top surface emission. The high reflectivity of the substrate side DBR was assured by the growth of 24-27 pairs of GaAs/AlAs quarter wavelength layers. The active regions were designed as resonant periodic gain structure i.e., the QW were located in subsequent antinode positions of the resonant standing wave. Different approaches were applied in VCSEL - the 3 sets of 3 QWs were separated by half wavelength thick GaAs spacers. In VECSEL the single QW (SQW) were grown in six subsequent antinode positions. The substantial difference between those heterostructures was: the VCSEL heterostructure was terminated with a high reflective $97 \%$ DBR of 14.5 pairs of GaAs/AlAs layers, and the VECSEL was terminated just by a single AlGaAs, $x=0.5$ window layer and GaAs protective cup. The window layer is there just in order to protect against the surface recombination of the carriers. The VECSEL cavity was enclosed by a concave semitransparent mirror with $f=50 \mathrm{~mm}$ and output coupling $5 \%$. When excited both devices were operated under threshold.

The transverse extent of the optical cavity was defined by the diameter of the laser beam and exceeded $100 \mu \mathrm{m}$. The spectral shape and the intensity of VCSEL emission and decay of the emission as a function of pump pulse energy were measured (Fig. 12). At low excitation densities the regular photoluminescence signal was observed. For higher excitations we have observed a clear threshold behavior and rapid increase in the emission intensity accompanied by the characteristic line 
narrowing. For very high pumping pulse energies $(\approx 2.67 \mathrm{~nJ})$ emission starts to behave unstable and shifts to lower energies, which is the result of heating. The plot of emission intensity from VCSEL structure vs. pump pulse energy is shown in Fig. 12. The threshold occurs at pump pulse energy of about $0.7 \mathrm{~nJ}$. At the same time a marked decrease in the carrier lifetime, from about $700 \mathrm{ps}$ to $50 \mathrm{ps}$, i.e., more than one order of magnitude has been observed. The above is a behavior characteristic of the transition from spontaneous to stimulated emission, and because it coincides with other characteristic features such as threshold in the emission intensity characteristics and line narrowing, it can be regarded as a final proof of the laser action in the investigated VCSEL structures.
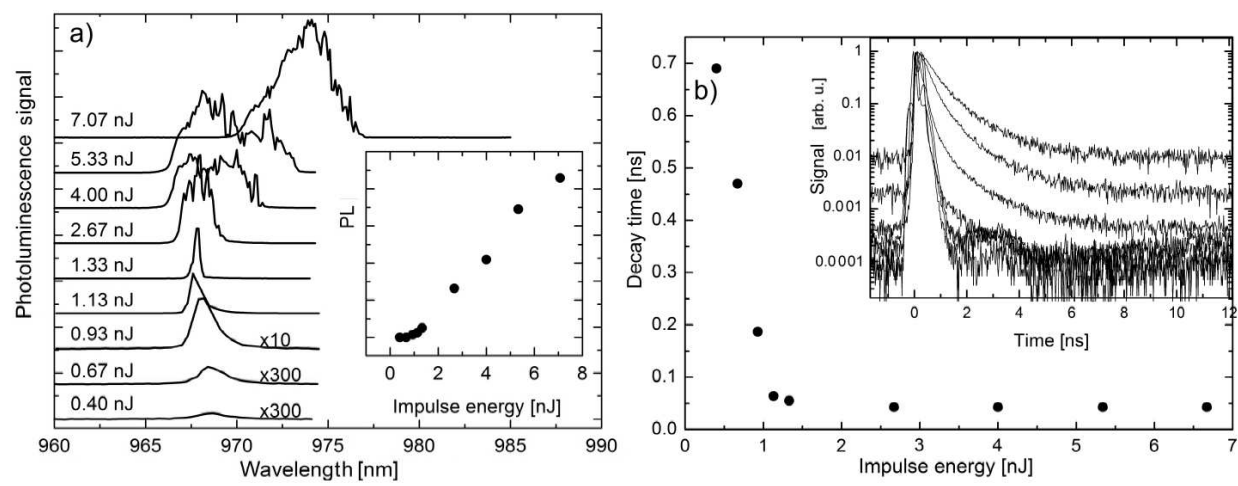

Fig. 12. VCSEL at high optical excitation. The threshold type increase of the integrated power emission (a) and decrease of the emission lifetime (b) are the evidence of the stimulated emission. The line are for the eye guide only. In the inserts the PL spectra and time decays are shown respectively.

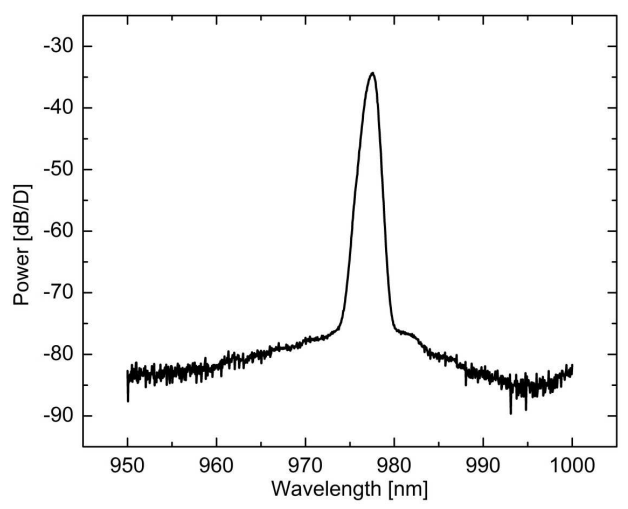

Fig. 13. VECSEL emission above threshold [33].

Similar experiment was carried with the VECSEL heterostructure. There at high power excitation the spectral shrinkage of the emission was observed (Fig. 13). 
Here due to the adjustable external mirror the laser operated on single lateral mode.

The pulse operation of the optical pumping was dictated by poor heat management. The over $100 \mu \mathrm{m}$ in diameter spots of high power excitation result in the rise temperature of the chip surface. This because of rather low characteristic temperature of those devices $T_{0} \approx 60 \mathrm{~K}$ results in the stimulated emission switch off. The proper heat management requires active cooling of the submount to which the laser chip is soldered. The chip itself has to have the substrate thinned down much below $100 \mu \mathrm{m}$ or totally removed. Also from the front surface heat should be efficiently extracted for instance by a diamond heat spreader.

Although both devices VCSEL and VECSEL can be operated in high output power mode under optical pumping the extended cavity of the latter one offers substantial advantages. The access to the laser cavity allows for integration with nonlinear optical element. The high amplitude of the electromagnetic field inside the cavity makes the frequency doubling especially efficient. The optical setups of VECSELs are very often expanded with other optical elements. Among them the semiconductor saturable absorber mirror (SESAM).

\section{SESAM}

The semiconductor saturable absorber mirror is a passive device which allows for laser mode-locking and generation of ultrashort impulses in femtosecond range $[30,31]$. Such short pulses cannot be generated by active devices. Only a passive shutter is fast enough to shape and stabilize the impulses in sub-picosecond range. The passive mode-locking makes that all the pulses are the copies of a single pulse traveling back and forth in the cavity. This makes that pulse-to-pulse variations are minimized and the phase of the pulses is constant. The main device parameters are center operation wavelength, modulation depth, saturation fluence, nonsaturable losses, and absorber recovery time. Those parameters can be designed and realized in epitaxial process.

The SESAM is a high reflectivity mirror integrated with an absorber whose absorption depends on the light intensity. We have fabricated a SESAM heterostructure using MBE. The heterostructure consisted of a DBR of high reflectivity - 27 pairs of GaAs/AlAs grown on GaAs substrate with an absorber, a single InGaAs/GaAs QW located in the top most quarter wavelength layer. The quarter wavelength layers were designed for maximum reflectivity at $1050 \mathrm{~nm}$. The cavity design was an antiresonant one. The antiresonant means that the GaAs slab does not constitute an optical cavity, there is no field build-up. Such composition reduces the light penetration into heterostructure thus the group delay dispersion (GDD). The absorption on the QW consists of saturable and unsaturable parts. Since too high unsaturable absorption deteriorates the laser parameters and a moderate amount of the saturable absorption is enough to drive the pulse width down to 100 fs range [30], the SQW was located outside the antinode position. 
The heterostructure was grown by MBE. There are substantial differences in epitaxial growth of the SESAM in comparison with all other devices discussed above. The SESAM is only one which requires the fast recombination of the carriers. The saturable absorption has to recover in round trip travel time of a pulse in the laser cavity. This time is usually of the range of picoseconds. Special growth procedures are required in order to decrease the carrier lifetime to such low value. The growth at low temperature at $200-300^{\circ} \mathrm{C}$ instead of standard $500^{\circ} \mathrm{C}$ intrusions of nonradiative defects thus promotes the nonradiative recombination. Also placing of the InGaAs QW just below the surface permits for the carrier recombination through the surface states. However, the intentional introduction of the defects in the QW must not destroy the total crystal perfectness. The ultrahigh reflectivity of the DBR and i.e., low nonsaturable losses are strictly required for proper laser operation.

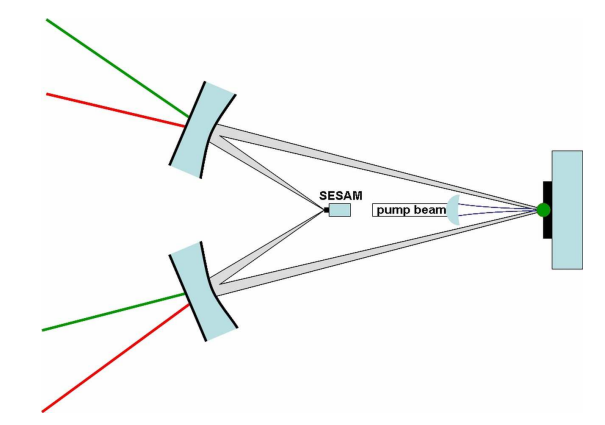

Fig. 14. The bidirectional double-V VECSEL based setup of pulse laser [32].

This SESAM was tested in nonstandard setup with a VECSEL as a gain medium. The bidirectional double $\mathrm{V}$ cavity permitted to emit two pulses (Fig. 14) [32]. In this device the pulse length was as short as $1 \mathrm{ps,} \mathrm{which} \mathrm{is} \mathrm{a}$ very good value in this class of devices [31]. The repetition rate was $0.758 \mathrm{GHz}$. This is a consequence of a total cavity length of $395 \mathrm{~mm}$.

\section{Conclusions}

We have presented a number of devices which profit from one-dimensional modulation of the refractive index. Such periodic modulation which is a basic feature of the photonic crystal permitted to freely design the features of those devices. The heterostructures of all those devices consisted of a cavity enclosed by two mirrors. By simply changing the reflective indices of the mirrors that is the number of high/low index layer pairs, we can switch from the spontaneous emission of RC LED to the stimulated one of the VCSEL (or VECSEL). The SESAM structure is like the VECSEL one. Both gain from the ultrahigh reflectivity of DBR. The difference lies in the QW material features. The long carrier lifetime is desired for VECSEL population inversion build-up needed for stimulated emission, 
whereas for SESAM the opposite is true, i.e. the shortest possible carrier lifetime is necessary.

\section{References}

[1] M.A. Aframowitz, Solid State Commun. 15, 59 (1974).

[2] Pochi Yeh, Optical Waves in Layered Media, Wiley, New York 1988.

[3] L.A. Coldren, S.W. Corzine, Diode Lasers and Photonic Integrated Circuits, Wiley, 1995.

[4] T.E. Sale, Vertical Cavity Surface Emitting Lasers, Research Studies Press Ltd, Wiley, 1995.

[5] Vertical-Cavity Surface-Emitting Laser, Eds. C. Wilmasen, H. Tempkin, L.A. Coldren, Cambridge University Press, Cambridge 1999.

[6] J. Muszalski, Semiconductor Microcavities, ITE, Warsaw 2003.

[7] M.S. Unlu, S. Strite, J. Appl. Phys. 78, 607 (1995).

[8] I. Kimukin, N. Biyikli, B. Butun, O. Aytur, S.M. Unlu, E. Ozbay, IEEE Phot. Technol. Lett. 14, 366 (2002).

[9] J. Kaniewski, J. Muszalski, J. Piotrowski, Phys. Status Solidi A 201, 2 (2004).

[10] A.J. Spring-Thorpe, T.P. Humphreys, A. Majeed, W.T. Moore, Appl. Phys. Lett. 55, 2138 (1989).

[11] J. Muszalski, T. Ochalski, M. Bugajski, XXVII International School on Physics of Semiconducting Compounds "Jaszowiec 99", 1999, unpublished results.

[12] J. Muszalski, Thin Solid Films 367, 299 (2000).

[13] H. Benisty, H. De Neve, C. Weisbuch, IEEE J. Quantum Electron. 34, 1612, 1632 (1998).

[14] E.F. Schubert, Y.-H. Wang, A.Y. Cho, L.-W. Tu, G.J. Zydzik, Appl. Phys. Lett. 60, 921 (1992).

[15] D. Delbeke, R. Bockstaele, P. Bienstman, R. Baets, H. Banisty, IEEE J. Sel. Topic Quant. Elect. 8, 189 (2002).

[16] K. Regiński, J. Muszalski, M. Bugajski, T. Ochalski, J.M. Kubica, M. Zbroszczyk, J. Kạtcki, J. Ratajczak, Thin Solid Films 367, 290 (2000).

[17] J. Muszalski, M. Bugajski, T.J. Ochalski, B. Mroziewicz, H. Wrzesinska, M. Gorska, J. Katcki, SPIE-Int. Soc. Opt. Eng. Proc. SPIE - The International Society for Optical Engineering 5230, 98 (2003).

[18] M. Bugajski, J. Muszalski, T. Ochalski, J. Kạtcki, B. Mroziewicz, Acta Phys. Pol. A 101, 105 (2002).

[19] A. Wójcik, T.J. Ochalski, J. Muszalski, E. Kowalczyk, K. Goszczyński, M. Bugajski, Thin Solid Films 412, 114 (2002).

[20] A.R. Pratt, T. Takamori, T. Kamijoh, J. Appl. Phys. 87, 8243 (2000).

[21] C. Dill, R.P. Stanley, U. Oesterle, D. Ochoa, M. Ilegems, Appl. Phys. Lett. 73, 3812 (1998).

[22] D.H. Lim, G.M. Yang, K.Y. Lim, J. Appl. Phys. 83, 2282 (1998).

[23] G.M. Yang, M.H. MacDougal, H. Zhao, P.D. Dapkus, J. Appl. Phys. 78, 3605 (1995). 
[24] P. Royo, R.P. Stanley, M. Ilegems, K. Streubel, K.H. Gulden, Appl. Phys. Lett. 77, 3899 (2000).

[25] K. Choquette, H.Q. Hou, Proc. IEEE 85, 1730 (1997).

[26] J.L. Jawell, J.P. Harbison, A. Scherer, Y.H. Lee, L.T. Florez, IEEE J. Quantum Electron. 27, 1332 (1991).

[27] T.E. Sale, Vertical Cavity Surface Emitting Lasers, Wiley, New York 1995.

[28] M. Kuznetsov, F. Hakimi, R. Sprague, A. Mooradian, IEEE J. Sel. Topics Quantum Electron. 5, 561 (1999).

[29] A.C. Tropper, S. Hoogland, Progr. Quantum Electron. 30, 1 (2006).

[30] I.D. Jung, F.X. Kaertner, N. Matuschek, D.H. Sutter, F. Morier-Genoud, Z. Shi, V. Scheuer, M. Tilsch, T. Tschudi, U. Keller, App. Phys. B 65, 137 (1997).

[31] U. Keller, A.C. Tropper, Phys. Rep. 429, 67 (2006).

[32] T.J. Ochalski, A. de Burca, G. Huyet, J. Lyytikäinen, M. Guina, M. Pessa, A. Jasik, J. Muszalski, M. Bugajski, in: CLEO/QELS and PhAST 2008 San Jose (CA, USA) 2008.

[33] J. Muszalski, A. Jasik, K. Pierściński, D. Pierścińska, A. Wójcik-Jedlińska, M. Bugajski, T.J. Ochalski, A. de Burca, G. Huyet, in: Int. Conf. on Semiconductor Materials and Optics, 200\%, National Institute of Telecommunications, Warsaw, Poland. 